\title{
Succession of bacterial communities on carrion is independent of vertebrate scavengers
}

\author{
Cody R Dangerfield ${ }^{1}$, Ethan H Frehner ${ }^{1}$, Evan R Buechley ${ }^{1,2}$, Çağan H Şekercioğlu ${ }^{1}$, William J Brazelton ${ }^{\text {Corresp. } 1}$ \\ ${ }^{1}$ School of Biological Sciences, University of Utah, Salt Lake City, UT, USA \\ 2 HawkWatch International, Salt Lake City, UT, USA \\ Corresponding Author: William J Brazelton \\ Email address: william.brazelton@utah.edu
}

The decomposition of carrion is carried out by a suite of macro- and micro-organisms who interact with each other in a variety of ecological contexts. The ultimate result of carrion decomposition is the recycling of carbon and nutrients from the carrion back into the ecosystem. Exploring these ecological interactions among animals and microbes is a critical aspect of understanding the nutrient cycling of an ecosystem. Here we investigate the potential impacts that vertebrate scavenging may have on the microbial community of carrion. In this study, we placed seven juvenile domestic cow carcasses in the Grassy Mountain region of Utah, USA and collected tissue samples at periodic intervals. Using high-depth environmental sequencing of the 16S rRNA gene and camera trap data, we documented the microbial community shifts associated with decomposition and with vertebrate scavenger visitation. The remarkable scarcity of animals at our study site enabled us to examine natural carrion decomposition in the near absence of animal scavengers. Our results indicate that the microbial communities of carcasses that experienced large amounts of scavenging activity were not significantly different than those carcasses that observed very little scavenging activity. Rather, the microbial community shifts reflected changes in the stage of decomposition similar to other studies documenting the successional changes of carrion microbial communities. Our study suggests that microbial community succession on carrion follows consistent patterns that are largely unaffected by vertebrate scavenging. 
1

2 Succession of bacterial communities on carrion is independent of vertebrate 3 scavengers

4 Cody R. Dangerfield ${ }^{1}$, Ethan Frehner ${ }^{1}$, Evan Buechley ${ }^{12}$, Çăgan H. Şekercioğlu ${ }^{1}$, William J.

5 Brazelton ${ }^{1}$

$6{ }^{1}$ School of Biological Sciences, University of Utah, Salt Lake City, UT

$7 \quad{ }^{2}$ HawkWatch International, Salt Lake City, UT

8

9 Corresponding Author:

10 William J. Brazelton

11 School of Biological Sciences

12257 South 1400 East Rm 201

13 Salt Lake City, UT 84112-0840 USA

14 william.brazelton@utah.edu

15 


\section{Abstract}

The decomposition of carrion is carried out by a suite of macro- and micro-organisms who interact with each other in a variety of ecological contexts. The ultimate result of carrion decomposition is the recycling of carbon and nutrients from the carrion back into the ecosystem. Exploring these ecological interactions among animals and microbes is a critical aspect of understanding the nutrient cycling of an ecosystem. Here we investigate the potential impacts that vertebrate scavenging may have on the microbial community of carrion. In this study, we placed seven juvenile domestic cow carcasses in the Grassy Mountain region of Utah, USA and collected tissue samples at periodic intervals. Using high-depth environmental sequencing of the 16S rRNA gene and camera trap data, we documented the microbial community shifts associated with decomposition and with vertebrate scavenger visitation. The remarkable scarcity of animals at our study site enabled us to examine natural carrion decomposition in the near absence of animal scavengers. Our results indicate that the microbial communities of carcasses that experienced large amounts of scavenging activity were not significantly different than those carcasses that observed very little scavenging activity. Rather, the microbial community shifts reflected changes in the stage of decomposition similar to other studies documenting the successional changes of carrion microbial communities. Our study suggests that microbial community succession on carrion follows consistent patterns that are largely unaffected by vertebrate scavenging. 
36

37

38

39

40

41

42

\section{Introduction}

Carrion, or dead animal tissue, provides a nutrient-rich resource for a wide array of organisms. At the smallest scale, both geographically and in organisms affected, carrion contributes nutrients to soils via nutrient leaching, thereby affecting microbial communities in soil near the carcass (Howard, Duos, and Watson-Horzelski 2010; Parkinson et al. 2009;

Parmenter and MacMahon 2009). Scaling upwards, carrion can be seen more directly as a food source to the many necrophagous arthropods and vertebrate scavengers (Jordan et al. 2015).

Furthermore, larger-scale impacts of carrion have been well documented in the massive die-off of salmon and cicada, which lead to large increases in resources and nutrient availability that affect a myriad of organisms including microbes, plants, fungi, and vertebrates (Hocking and Reynolds 2011, 2012; Jordan et al. 2015; Tiegs et al. 2009, 2011; Yang 2004). The versatile methods by which carrion can be produced and consumed gives it the potential to impact many facets of an ecosystem, and the pathway by which it decomposes depends on the environmental conditions and the interactions that form among the organisms that compete over its resources. categorized into stages based on the descriptions in Payne (1965): fresh, bloat, active decay, advanced decay, and putrid dry remains. A body enters the fresh stage immediately after death, and depletion of internal oxygen triggers autolysis of the cells. Shortly after death, endogenous microbes begin to metabolize the body and produce volatile compounds. As the activity of these microbes fills the body cavity with gases, causing the carcass to distend, the carcass transitions into the bloat stage. Active decay follows the bloat stage when the body cavity ruptures, releasing the gases and allowing invertebrates to consume a larger portion of the soft tissue within the body cavity (Carter, Yellowlees, and Tibbett 2007). After the removal of most of the 
59 soft tissue, invertebrate activity decreases, and the carcass transitions into the advanced decay

60 stage. The carrion enters the putrid dry remains stage when the carcass has desiccated, leaving

61 only bones and small amounts of skin and hair (Goff 2009; Payne 1965). organisms (Hanski 1987; Janzen 1977; Wilson and Wolkovich 2011). Due to the high level of competition, the organisms that consume carrion have developed behaviors in order to monopolize the nutrients of carcass for themselves. These complex interactions among microbes and scavenging fauna, along with abiotic factors (e.g. precipitation and temperature), often impact the duration and occurrence of the previously described stages (Carter, Yellowlees, and Tibbett 2008, 2010; Comstock et al. 2015; Galloway, Jones, and Parks 1989; Payne 1965; Rozen, Engelmoer, and Smiseth 2008; Shukla et al. 2017). For example, some microbes that begin to metabolize the carrion soft tissue after an animal's death also produce toxins in order to hinder consumption from other organisms (Blandford et al. 2019; Burkepile et al. 2006; Janzen 1977). In response, some scavengers, such as the turkey vulture (Cathartes aura), have developed an unusually high tolerance to decomposer-produced toxins (e.g. botulinum toxin), and the highly acidic conditions present in their hindgut reduce the likelihood of microbes surviving consumption and infecting the vulture itself (Beasley, Olson, and DeVault 2015; DeVault, Rhodes Olin E., and Shivik 2003; Roggenbuck et al. 2014). Furthermore, behaviors such as the burial of carcasses have developed in both vertebrate and invertebrate scavengers in order to seclude the carrion from climatic conditions, microbes, and other scavengers to slow decomposition and secure the resources for themselves (Frehner et al. 2017; Rozen et al. 2008; Shukla et al. 2017). Burying beetles (Nicrophorus spp.) further suppress competition with 
82 Rozen et al. 2008; Shukla et al. 2017). In doing so, these beetles limit decomposition and can

83 delay the carcasses from entering the bloat or active decay stages (Shukla et al. 2017), which are

84 mainly dictated by microbial and insect activity (Finley, Benbow, and Javan 2015; Goff 2009;

85 Payne 1965).

Researching these interactions is important to understand how carrion decomposition impacts nutrient cycling and the importance that carrion has on ecosystems (Barton 2015; Barton et al. 2013). There are also forensic benefits to understanding these interactions as the pattern of succession on carrion and cadavers has the potential to determine postmortem time intervals (PMI; Amendt et al. 2011; Anderson 2015). Historically, a majority of studies focused on forensic entomology to determine PMI (Byrd and Allen 2001; Michaud and Moreau 2009; Payne 1965; Schoenly and Reid 1987). However, recent studies have used DNA sequencing technology to investigate the potential for these microbes to serve as PMI indicators (Burcham et al. 2016; Finley et al. 2015; Guo et al. 2016; Metcalf et al. 2013; Pechal et al. 2014) and further characterize the microbiome carrion (Hyde et al. 2013; Pechal et al. 2013, 2018, 2019;

Weatherbee, Pechal, and Benbow 2017). These studies have further characterized the microbial communities associated with carrion decomposition and how seasonal changes and macroinvertebrates impact those microbial communities. focused on scavenger avoidance of microbe-laden carrion (Blandford et al. 2019; Burkepile et al. 2006). It has long been suggested that scavengers may facilitate the spread of pathogens throughout an ecosystem (Houston and Cooper 1975), but scavengers' involvement in disease 103 ecology is complex and requires further study (Beasley et al. 2015). A recent study has suggested that macroinvertebrates feeding on carrion may act as vectors of microbes among carcasses 
105 (Pechal et al. 2019), but to our knowledge, no study has investigated the influence of vertebrate

106 scavengers, or the lack thereof, on the microbial communities of carrion in an terrestrial

107 environment. In this study, we used environmental DNA sequencing and vertebrate scavenging

108 data to investigate decomposition dynamics and potential impacts that vertebrate scavengers

109 have on the microbiome of carrion in the Great Basin Desert of Utah. Specifically, we tested the

110 hypothesis that vertebrate scavengers disrupt the typical progression of carcass decomposition

111 and introduce variability into the succession of microbial communities on carrion.

\section{Methods}

\section{Study sites and field data collection}

In this study, we investigate the bacterial communities of bovine carcasses in the Grassy Mountain region of Utah, USA $\left(40.87^{\circ} \mathrm{N},-113.03^{\circ} \mathrm{W}\right)$ from May to June, 2015 . To do so, we experimentally placed juvenile domestic cow (Bos taurus) carcasses $(n=7)$ in the study site and monitored their decomposition using camera traps to identify vertebrate scavenger activity and by collecting tissue samples at regular intervals to identify progression of microbial communities. We collected tissue samples from each of the carcasses during five sampling periods (Day 1, Day 4, Day 12, Day 18, and Day 26) (Figure 1). The calves were obtained from one local Utah dairy and had died from natural causes either during or shortly after birth. The carcasses were collected on the day of birth/death, and were kept frozen for 1-6 months until their placement in the field. Carcasses thawed during transport through the desert but were still cold at the time of final placement and of the first sample collection (i.e. Day 1). They were placed at least $3 \mathrm{~km}$ apart and fixed to a concealed stake in the ground to prevent scavengers from removing the complete carcass. All carcasses were placed and sampled on the same day. 
127 The carcasses weighed between 18.6 and $26.9 \mathrm{~kg}$. Carcasses were placed on sites that included

128 sparse Utah juniper (Juniperus osteosperma), greasewood (Sarcobatus vermiculatus), and widely

129 distributed cheatgrass (Bromus tectorum). The soil in the study area is composed of loose to

130 moderately compacted limnological sediments, including gravels and clays. The study area is

131 arid and largely homogenous. Study area temperatures varied between $7-40^{\circ} \mathrm{C}$, and there was no

132 precipitation during the experiment. Field methods were approved by the University of Utah

133 Institutional Animal Care and Use Committee (Protocol Number 15-06001) and by the US

134 Bureau of Land Management through a joint letter of agreement.

to monitor vertebrate scavenging activity. The cameras were programmed to take 1 photo when

137 triggered, with a 10-s delay between subsequent photos to reduce saturation of photos from the

138 same animal visitation event. All photos collected over the course of the study were entered into

139 CameraBase Version 1.7 (Tobler 2007), a camera-trap photo management platform in Microsoft Access.

140 We analyzed each of these photos individually and identified any vertebrates present in the photos to

141 species. We identified arrival times after carcass placement and duration of presence at carcass for each

142 scavenger species.

Tissue samples $\left(\sim 15 \mathrm{~cm}^{3}\right.$ in size $)$ were excised from the hind leg of each carcass. Samples contained muscle, skin, fat, and hair during the early stages of decomposition, and skin and hair in the latter stages of decomposition when no soft tissue remained. Soil samples directly adjacent to where the carcass was placed were acquired during the first sampling period for two of the seven sites. Soil samples for the remaining sites were taken $5 \mathrm{~m}$ from the carcass during the second sampling period due to concerns that the earlier soil samples were collected in too close 
150 microbial diversity results indicated that all soil samples were very similar to each other,

151 alleviating this concern. DNA was extracted from all carcass and soil samples using the

152 PowerSoil DNA Isolation Kit (MO BIO Laboratories, Carlsbad, CA, USA) according to the

153 manufacturer's instructions and stored at $-20^{\circ} \mathrm{C}$.

\section{Bacterial 16S rRNA gene sequencing}

155

156

157

158

159

160

161

162

163

164

165

166

167

168

169

170

171
The samples were submitted to the Michigan State University genomics core facility for bacterial 16S rRNA gene amplicon sequencing. The V4 region of the 16S rRNA gene (defined by primers $515 \mathrm{~F} / 806 \mathrm{R}$ ) was amplified with dual-indexed Illumina fusion primers as described by Kozich et al. (2013). Amplicon concentrations were normalized and pooled using an Invitrogen SequalPrep DNA Normalization Plate. After library QC and quantitation, the pool was loaded on an Illumina MiSeq v2 flow cell and sequenced using a standard 500 cycle reagent kit. Base calling was performed by Illumina Real-Time Analysis (RTA) software v1.18.54. Output of RTA was demultiplexed and converted to fastq files using Illumina Bcl2fastq v1.8.4. Paired-end sequences were filtered and merged with each other (to form, on average, $253 \mathrm{bp}$ merged sequences) with USEARCH 8 (Edgar 2010), and additional quality filtering was conducted with the mothur software platform (Schloss et al. 2009) to remove any sequences with ambiguous bases and more than 8 homopolymers. Chimeras were removed with mothur's implementation of UCHIME (Edgar et al. 2011). The sequences were pre-clustered with the mothur command pre.cluster (diffs $=1$ ), which reduced the number of unique sequences from 1,136,609 to 784,953 . This pre-clustering step removes rare sequences most likely created by sequencing errors (Schloss and Westcott 2011).

\section{Bacterial diversity analyses}

PeerJ reviewing PDF | (2019:08:40564:2:0:NEW 27 Apr 2020) 
The unique, pre-clustered sequences were considered to be the operational taxonomic

173 units (OTUs) for this study and formed the basis of all alpha and beta diversity analyses, as in

174 our previous study (Dangerfield, Nadkarni, and Brazelton 2017). Sequence reads were not

175 rarefied for alpha diversity and evenness calculations because there was no correlation between

176 diversity indices and sequencing depth for this study (Table S1). Taxonomic classification of all

177 sequences was performed with mothur using the SILVA reference alignment (SSURefv123) and

178 taxonomy outline (Pruesse, Peplies, and Glöckner 2012). Taxonomic counts generated by mothur

179 and edgeR were visualized using the R package phyloseq 1.20.0 (McMurdie and Holmes 2013).

\section{Statistical analyses}

Alpha diversity and evenness were calculated with the Shannon, invsimpson, and simpsoneven calculators provided in the mothur package (Schloss et al. 2009). Differences between alpha diversities and evenness were tested for significance using the Dunnett-Tukey-

Kramer test, which accounts for multiple comparisons among samples with unequal sizes and variances (Lau 2013). Beta diversity was measured using the Morista-Horn biodiversity index, as implemented in mothur. This index was chosen because it reflects differences in the abundances of shared OTUs without being skewed by unequal numbers of sequences among samples.

Differences between community compositions were tested for significance using AMOVA (analysis of molecular variance) as implemented in mothur (Pruesse et al. 2012). Morisita-Horn community dissimilarity among samples was visualized using a nonmetric multidimensional scaling (NMDS) plot. This plot was generated using the ordinate and plot ordination commands

192 in phyloseq (McMurdie and Holmes 2013). The ggplot2 function stat_ellipse was added to draw 193 95\% confidence level ellipses (assuming t-distribution) in the NMDS plot (Wickham 2016).

194 Environmental variables (temperature, scavenger counts, and scavenging duration) were fitted to 
195 the community composition ordination with the envfit function in the vegan package (Oksanen et

196 al. 2019). Differences in the relative abundance of OTUs between stages was measured using the

197 R package edgeR (Robinson, McCarthy, and Smyth 2009) as recommended by McMurdie and

198 Holmes (2014). The differential abundance of an OTU (as measured in units of log2 fold change

199 by edgeR) was considered to be statistically significant if it passed a false discovery rate

200 threshold of 0.05 . Taxa were determined characteristic to that specific stage if they were found

201 differentially abundant in one stage compared to all other stages and soil. These taxa are referred

202 to as "characteristic taxa" for the purposes of this paper. To investigate potential environmental

203 contamination of carcass samples, OTUs with at least 20 sequence counts among all samples

204 were assigned to either fresh carcass or soil using the sink-source Bayesian approach of

205 SourceTracker2 v2.0.1 (Knights et al. 2011) with rarefying to 66,001 sequences for sinks and

20616,828 sequences for sources. Similar results were obtained without rarefying sequence counts

207 (Figure S1, S2). The one carcass sample that was determined to be contaminated by soil via

208 SourceTracker2 was excluded from alpha and beta diversity analyses.

209 Data Availability

210 All sequence data are publicly available at the NCBI Sequence Read Archive under BioProject

211 PRJNA525153.

212

213 Results

214 Carcass decomposition

215

Sampling periods were categorized into stages of decomposition based on physical

216 interpretation of the carcasses (Figure 1) as determined from camera trap photographs taken of

217 each carcass and as described by Payne (1965). Day 1 was determined to be "fresh", as the 
218 carcasses were kept frozen promptly after death. The carcasses was determined to be in the

219 "bloat" stage at Day 4, as evidenced by the body cavity becoming distended by gases emitted

220 during microbial decomposition. The bloat stage may have begun earlier than Day 4, but was not

221 apparent in the camera trap photographs. The later sampling periods (Day 12, 18, and 26) were

222 all categorized as the "active decay" stage, because of the large decrease in carcass size and the

223 presence of skin tissue on the carcass.

Vertebrate scavengers that fed at the carcasses included American badger (Taxida taxus),

common raven (Corvus corax), coyote (Canis latrans), kit fox (Vulpes macrotis), turkey vulture,

frequent scavenger to feed at the carcasses, and the majority of vertebrate scavenging occurred between Day 4 and Day 12 of the study (Table 1).

\section{Impact of scavenging on bacterial communities}

compared Morisita-Horn dissimilarities between high- and low-scavenging sites directly after the experienced $88 \%$ of the total scavenging duration observed during the study (Table 1 ). sites were minimal and not significant $\left(A M O V A F_{s}=0.603245, p=0.636\right)$. Additionally, fitting

237 of scavenging parameters (individuals per week and scavenging duration per week) to the

238 NMDS ordination (Figure 2) yielded no significant correlations. 
To identify individual operational taxonomic units (OTUs) that scavengers may have

240

241

242

243 244 sites.

245

246

247

248

249

250

251

252

253

254

255

256

257

258

259

260

introduced to the carcasses, we contrasted the relative abundances of OTUs in high-scavenging

sites to low-scavenging sites directly after the major scavenging events (Day 12 samples). This

comparison yielded 39 OTUs that were differentially abundant in the two high-scavenging sites

in comparison to low-scavenging sites. Only a few OTUs were present in both high-scavenging

We also examined the relative abundance patterns of OTUs classified as genera reported by previous studies to be associated with macroinvertebrates (Debruyn 2018; Dharne et al. 2008;

Gupta et al. 2011, 2014; Lee et al. 2014; Shukla et al. 2017; Tóth et al. 2008; Weatherbee et al. 2017). All macroinvertebrate-associated genera reach peak relative abundances during the later sampling periods except for Providencia and Myroides (Figure 3).

\section{Bacterial community changes over time}

Microbial community differences are visualized in Figure 2, where each data point represents the overall bacterial community composition of one sample and the distance between points represents the dissimilarity between samples. There are two primary shifts in community composition: one from Day 1 to Day 4 and a second from Day 4 to later sampling periods (Figure 2). The 95\% confidence ellipses show consistent separation between these sampling periods. The only exception to this pattern was a single sample from Day 4 that clusters with soil samples in Figure 2. We examined the bacterial community composition of this outlier sample in more detail with SourceTracker2 (Knights et al. 2011), which revealed that $83 \%$ of OTUs in the outlier sample could be confidently assigned to soil. Therefore, we concluded that this 
261 sample had been contaminated with soil during sampling and/or handling, and we excluded this

262 sample from all downstream analyses.

263

264

265

266

267

268

269

270

271

272

273

274

275

276

277

278

279

280

281

282

283

Figure 2 also shows that the bacterial community composition of all carcasses at later sampling dates (Days 12, 18, 26) are highly similar and not significantly different from each other. The patterns visualized in the NMDS ordination were tested with an AMOVA that confirmed significant differences between the Day 1, Day 4, and later sampling periods (Table

2). These three clusters of bacterial community composition (Day 1, Day 4, and Days 12-18-26) correspond to the three stages of decomposition identified by physical interpretation of the carcasses (Day 1 = "fresh", Day 4 = "bloat", and Days 12-18-16 = "active decay").

Proteobacteria was the most common phylum in the fresh stage, accounting for $48 \%$ of microbial community composition, decreasing to $11 \%$ for both the bloat and active decay stages (Figure 4). Conversely, Firmicutes abundance increased as decomposition progressed. Firmicutes increased from $31 \%$ in the fresh stage to $72 \%$ and $84 \%$ of the microbial community in bloat and active decay, respectively. Moraxellaceae represented 30\% of the total abundance of the fresh stage (Figure 4), whereas Moraxellaceae only accounted for $2 \%$ and $0.8 \%$ of the total abundance in bloat stage and active decay, respectively. By contrast, Clostridia was dominant in the bloat stage, accounting for $70 \%$ of the total abundance, and accounting for only $3 \%$ of total abundance in the fresh stage (Supplementary Krona files).

\section{Taxa characteristic to each stage of decomposition}

The taxonomic classifications (at order and family level) of all OTUs identified as characteristic to each stage of decomposition (as defined by differential abundance analysis described in Methods) are shown in Figure 4. Each bar in Figure 4 represents the total 
284 community composition of each stage, in which "Ubiquitous taxa" represents taxa that are

285 equally abundant across two or more groups and the remaining taxa in each bar represent OTUs

286 that are characteristic to that stage. There were 323 OTUs that were characteristic to the fresh

287 stage, and these taxa accounted for $\sim 62 \%$ of the total abundance in that stage. These fresh stage

288 taxa are dominated by Moraxellaceae, Flavobacteriaceae, Pseudoalteromonadaceae,

289 Planococcaceae, Staphylococcaceae, and an unclassified Bacillales family. The bloat stage

290 contained fewer characteristic taxa (106 OTUs) than the fresh stage, and these taxa accounted for

$291 \sim 56 \%$ of the total abundance in that stage. The characteristic bloat taxa mainly consisted of

292 Clostridia OTUs. In particular, five Clostridia OTUs accounted for $49 \%$ of the bloat stage's

293 total abundance. The active decay stage contained 230 characteristic OTUs that accounted for

$294 \sim 64$ percent of the total abundance. Active decay still contained OTUs from Clostridia, but these

295 OTUs are different than those Clostridia OTUs in the bloat stage and account for less of the total

296 abundance. Active decay stage contained other prominent characteristic taxa from

297 Flavobacteriaceae, Enterococcaceae, Xanthomonadales and an unclassified Bacillales family. All

298 characteristic OTUs, their proportional abundances, and taxonomic classifications are reported in 299 the Supplemental Information.

\section{Alpha diversity}

Figure 5 reports the OTU Shannon diversity index of each stage of decomposition. Alpha diversity was highest during the fresh stage (Shannon diversity range $=4.8-7.2$ ) and decreased during the bloat stage (Shannon diversity range $=3.3-4.4$ ). Diversity was more variable but greater on average in the active decay stage (Shannon diversity range $=3.8-6.7$ ). The shifts in 
307 passed a Dunnett-Tukey-Kramer significance test. Similar patterns were also evident with the 308 Inverse Simpson and Evenness (from Simpson) indices.

\section{Discussion}

\section{No correlation between vertebrate scavenging and bacterial community composition}

Carrion is a valuable resource for which many organisms compete, including vertebrate scavengers, macroinvertebrates, and microbes. Although a few studies have documented the association of bacteria with macroinvertebrates on carrion (Pechal et al. 2013, 2019; Rozen et al. 2008; Shukla et al. 2017), this is the first study to investigate the impact of vertebrate scavengers on the bacterial community of carrion in a terrestrial environment. Our study site in an arid region of Utah was well-suited for this experiment because of its unusually sparse vertebrate scavenging activity (Frehner et al., In Review). Studying the succession of bacterial communities in the absence of vertebrate impacts would have been difficult or impossible in a typical environment without significant experimental manipulations.

One potential consequence of vertebrate scavenging could be the disruption of the progression of carcass decomposition, introducing variability into the succession of microbial communities. Our results did not provide any support for this hypothesis. No impact of scavenging activity on the bacterial community composition of carrion was observed. Overall, the bacterial communities of carcasses that experienced vigorous vertebrate scavenging (e.g. 15 individuals and 3.2 hours of activity) were highly similar to the bacterial communities of carcasses at the same stage of decomposition but with almost no vertebrate scavenging (e.g. 1 individual and 1 minute of activity). 
(OTUs) in this study significantly shifted in abundance after the peak in scavenging activity on

particularly abundant in both high-scavenging sites. If the impact of scavenging on microbial composition is rapid and transient, it may not have been captured by our sampling density of points (Benbow and Pechal (2017).

Although we saw no evidence of bacterial taxa consistently associated with vertebrate scavenging, we did find taxa that have been previously associated with turkey vultures (Roggenbuck et al. 2014). However, the abundances of these taxa were not higher in sites that experienced more scavenging by turkey vultures. Furthermore, Roggenbuck et al. (2014) speculated that these taxa are most likely derived not from the turkey vultures, but from the carrion they consume.

\section{Bacterial taxa associated with macroinvertebrates}

Although our study was not designed to investigate the impact of macroinvertebrates, we observed several genera of microbes that have been previously associated with scavenging 
352 consistent with other studies (Debruyn 2018; Shukla et al. 2017; Weatherbee et al. 2017). With

353 the exception of Providencia and Myriodes, most of these genera exhibited increased abundance

354 when the carcasses entered into active decay. This increase in macroinvertebrate-associated taxa

355 is consistent with the increases of macroinvertebrate activity that typically occur during the

356 active decay stage (Carter et al. 2007; Finley et al. 2015; Matuszewski et al. 2010; Payne 1965).

357 Unfortunately, we were not able to include observations of macroinvertebrates in our study 358 design.

\section{Stages of decomposition have consistent bacterial communities}

During this study, the carcasses exhibited three stages of decomposition (fresh, bloat, and active decay), and each stage was characterized by a unique community of bacteria. Although the weather, geography, and vertebrate scavenging activity of our study was notably different than in previous studies, our observed patterns of bacterial community succession are remarkably similar to those previously reported for carrion (Hyde et al. 2013; Pascual et al. 2017; Pechal et al. 2013, 2014, 2018; Weatherbee et al. 2017). Pechal et al. (2013) reported that Proteobacteria were dominant in the early stages of decomposition and declined as decomposition progressed and that Firmicutes abundances progressively increased in the later stages of decomposition.

Similarly, 8 of the 14 families that exhibited distinct temporal patterns during the decomposition process in Pascual et al. (2017) exhibited similar patterns in our study (Figure 4). aerobic/anaerobic microbial community shifts during the progression of decay (Burcham et al.

373 2016; Debruyn 2018; Finley et al. 2015; Goff 2009; Hyde et al. 2013; Metcalf et al. 2013;

374 Pascual et al. 2017; Pechal et al. 2013, 2014). Abundance patterns of OTUs from Moraxellaceae, 
375 all of which are known to be aerobic (Pascual et al. 2017), exemplify this pattern: they represent

$37630.3 \%$ of total abundance in the fresh stage, $2.4 \%$ in the bloat stage (when anaerobic Clostridia

377 and Enterobacteriaceae are dominant), and $0.78 \%$ in the active decay stage. Furthermore, the

378 dominance of anaerobic bacteria during the bloat stage is exemplified with Clostridia comprising

$37970 \%$ of the microbial community in the bloat stage (Dataset 2 ). This dominance of a few taxa

380 during the bloat stage is associated with low alpha diversity values (Figure 5), whereas Pascual et

381 al. (2017) observed the highest alpha diversity during the bloat stage (described as the

382 putrefaction stage) in forest ecosystems.

383 Most other studies observe more rapid progression through the stages of decomposition

384 (Pascual et al. 2017; Pechal et al. 2013, 2014). It is possible that carcasses during the last

385 sampling period of our study were in "advanced decay", but there was no discernible change in

386 tissue composition of the carcasses compared to the previous two sampling periods. The delay in

387 decomposition is most likely the result of climatic factors. Previous studies investigating carrion

388 microbial communities were conducted in areas with much higher humidity, whereas this study

389 experienced arid conditions and high temperatures during a period with no precipitation. Active

390 decay has been shown to be limited or hindered by hot and dry climates similar to the conditions

391 present in this study, resulting in partial mummification (Galloway et al. 1989). During this

392 process, carcasses develop a mummified shell over the skeleton as the skin desiccates while

393 macroinvertebrate activity continues underneath, in the body cavity. This partial mummification

394 may explain the prolonged intact composition of the carcass in the latter sampling periods

395 (Figure 1) associated with an increased abundance of macroinvertebrate-associated bacterial taxa

396 (Figure 3).

397 
398

399

400

401

402

403

404

405

406

407

408

409

410

411

412

413

414

415

416

417

418

419

\section{Conclusion}

In this study, we investigated microbial succession associated with decomposition of cow carcasses that experienced notably little vertebrate scavenging activity. Our results provided no support for the hypothesis that isolated, intense vertebrate scavenging events affect the progression of carcass decomposition or the bacterial communities on carrion. Instead, the bacterial community composition of all carcasses consistently reflected the stage of decomposition, regardless of vertebrate scavenging activity. A more expansive study with additional time points and additional replicate carcasses may have been able to detect more subtle shifts in the abundance of individual taxa in response to scavenging within each stage of decomposition.

Our results are remarkably similar to those of other studies conducted in wetter, milder conditions with greater vertebrate scavenging activity (Burcham et al. 2016; Hyde et al. 2013; Pascual et al. 2017; Pechal et al. 2013, 2014, 2019; Weatherbee et al. 2017), suggesting that bacterial community succession on carrion follows consistent patterns that are largely unaffected by many external factors. However, we observed differences in the timing of decomposition, most likely due to the arid climate, and perhaps associated with this, differences in the trajectory of microbial diversity through later stages of decomposition (Figure 5) compared with studies in wetter environments (e.g. Pascual et al., 2017).

Our results provide additional support for the use of microbial community composition as a reliable forensic indicator of the timing of carcass decomposition. In particular, this study was able to show that arid environmental conditions and variable activity of vertebrate scavengers did not greatly affect the overall succession of microbial communities on cow carcasses. 


\section{References}

422

423

424

425

426

427

428

429

430

431

432

433

434

435

436

437

438

439

440

Amendt, J., C. S. Richards, C. P. Campobasso, R. Zehner, and M. J. R. Hall. 2011. "Forensic Entomology : Applications and Limitations.” 379-92.

Anderson, Gail S. 2015. "Human Decomposition and Forensics.” Pp. 541-60 in Carrion Ecology, Evolution, and Their Applications.

Barnes, K. M., D. E. Gennard, and R. A. Dixon. 2010. “An Assessment of the Antibacterial Activity in Larval Excretion / Secretion of Four Species of Insects Recorded in Association with Corpses , Using Lucilia Sericata Meigen as the Marker Species.” Bulletin of Entomological Research (March 2010):635-40.

Barton, Philip S. 2015. “The Role of Carrion in Ecosystems.” Pp. 273-86 in Carrion Ecology, Evolution, and Their Applications.

Barton, Philip S., Saul A. Cunningham, David B. Lindenmayer, and Adrian D. Manning. 2013. "The Role of Carrion in Maintaining Biodiversity and Ecological Processes in Terrestrial Ecosystems." Oecologia 171(4):761-72.

Beasley, James C., Zach H. Olson, and Travis L. DeVault. 2015. "Ecological Role of Vertebrate Scavengers." in Carrion Ecology, Evolution, and Their Applications.

Benbow, M. Eric and Jennifer L. Pechal. 2017. “Approaches and Considerations for Forensic Microbiology Decomposition Research.” Pp. 56-71 in Forensic Microbiology, edited by D. O. Carter and J. K. Tomberlin.

Blandford, Makeely I., Mohammad Katouli, Ben L. Gilby, Christian O. Dea, Andrew D. Olds, 
and Thomas A. Schlacher. 2019. "Estuarine, Coastal and Shelf Science Not All Rotten Fish Stink : Microbial Changes in Decaying Carcasses Increase Cytotoxicity and Potential Risks to Animal Scavengers." Estuarine, Coastal and Shelf Science 227(December 2018):106350.

Burcham, Z. M., J. A. Hood, J. L. Pechal, K. L. Krausz, J. L. Bose, C. J. Schmidt, M. E. Benbow, and H. R. Jordan. 2016. "Fluorescently Labeled Bacteria Provide Insight on PostMortem Microbial Transmigration.” Forensic Science International 264:63-69.

Burkepile, Deron, John D Parker, Clifton Woodson, Heath Mills, Julia Kubanek, Patricia Sobecky, and Mark Hay. 2006. Chemically Mediated Competition between Microbes and Animals: Microbes as Consumers in Food Webs. Vol. 87.

Byrd, Jason H. and Jon C. Allen. 2001. "The Development of the Black Blow Fly, Phormia Regina (Meigen).” Forensic Science International 120(1-2):79-88.

Carter, David O., David Yellowlees, and Mark Tibbett. 2007. "Cadaver Decomposition in Terrestrial Ecosystems." Naturwissenschaften 12-24.

Carter, David O., David Yellowlees, and Mark Tibbett. 2008. "Temperature Affects Microbial Decomposition of Cadavers (Rattus Rattus) in Contrasting Soils.” Applied Soil Ecology 40(1):129-37.

Carter, David O., David Yellowlees, and Mark Tibbett. 2010. "Moisture Can Be the Dominant Environmental Parameter Governing Cadaver Decomposition in Soil." Forensic Science International 200(1):60-66.

Comstock, Jenna L., Jean Paul Desaulniers, Hélène N. LeBlanc, and Shari L. Forbes. 2015. "New Decomposition Stages to Describe Scenarios Involving the Partial and Complete 
Dangerfield, Cody R., Nalini M. Nadkarni, and William J. Brazelton. 2017. “Canopy Soil Bacterial Communities Altered by Severing Host Tree Limbs.” PeerJ 5:e3773.

Debruyn, Jennifer Mary. 2018. “Temporal and Spatial Impact of Human Cadaver Decomposition on Soil Bacterial and Arthropod Community Structure and Function.” 8(January):1-12.

DeVault, Travis L., Jr. Rhodes Olin E., and John A. Shivik. 2003. "Scavenging by Vertebrates: Behavioral, Ecological, and Evolutionary Perspectives on an Important Energy Transfer Pathway in Terrestrial Ecosystems.” Oikos 102(2):225-34.

Dharne, M. S., A. K. Gupta, A. Y. Rangrez, H. V. Ghate, M. S. Patole, and Y. S. Shouche. 2008. “Antibacterial Activities of Multi Drug Resistant Myroides Odoratimimus Bacteria Isolated from Adult Flesh Flies (Diptera: Sarcophagidae) Are Independent of Metallo BetaLactamase Gene.” Brazilian Journal of Microbiology 39(2):397-404.

Edgar, RC. 2010. "Search and Clustering Orders of Magnitude Faster than BLAST." Bioinformatics 26(19):2460-61.

Edgar, Robert C., Brian J. Haas, Jose C. Clemente, Christopher Quince, and Rob Knight. 2011. “UCHIME Improves Sensitivity and Speed of Chimera Detection.” Bioinformatics 27(16):2194.

Finley, Sheree J., M. Eric Benbow, and Gulnaz T. Javan. 2015. "Microbial Communities 480 Associated with Human Decomposition and Their Potential Use as Postmortem Clocks." International Journal of Legal Medicine 129(3):623-32. 
483

484

485

486

487

488

489

490

491

492

493

494

495

496

497

498

499

500

501

502

503

“Subterranean Caching of Domestic Cow (Bostaurus) Carcasses by American Badgers

(Taxidea Taxus ) in the Great Basin Desert, Utah.” Western North American Naturalist 77(1):124-29.

Frehner, Ethan H., Evan R. Buechley, and Çağan H. Şekercioğlu. In Review. "Season Mediates Vertebrate Scavenging Rates in the Great Basin Desert." Ecosphere.

Galloway, Alison, Allen Jones, and Bruce Parks. 1989. "Decay Rates of Human Remains in an Arid Environment." Journal of Forensic Sciences 34(3):607-16.

Goff, M. Lee. 2009. “Early Post-Mortem Changes and Stages of Decomposition in Exposed Cadavers.” Experimental and Applied Acarology 49(1-2):21-36.

Guo, Juanjuan, Xiaoliang Fu, Huidan Liao, Zhenyu Hu, Lingling Long, Weitao Yan, Yanjun Ding, Lagabaiyila Zha, Yadong Guo, Jie Yan, Yunfeng Chang, and Jifeng Cai. 2016. "Potential Use of Bacterial Community Succession for Estimating Post-Mortem Interval as Revealed by High-Throughput Sequencing." Scientific Reports 6(April):1-11.

Gupta, A. K., G. Rastogi, D. Nayduch, S. S. Sawant, R. R. Bhonde, and Y. S. Shouche. 2014. "Molecular Phylogenetic Profiling of Gut-Associated Bacteria in Larvae and Adults of Flesh Flies." Medical and Veterinary Entomology 28(4):345-54.

Gupta, Arvind Kumar, Mahesh Shantappa Dharne, Ashraf Yusuf Rangrez, Pankaj Verma, Hemant V. Ghate, Manfred Rohde, Milind Shivaji Patole, and Yogesh Shreepad Shouche. 2011. "Ignatzschineria Indica Sp. Nov. and Ignatzschineria Ureiclastica Sp. Nov., Isolated from Adult Flesh Flies (Diptera: Sarcophagidae).” International Journal of Systematic and Evolutionary Microbiology 61(6):1360-69. 
504 Hanski, Ilkka. 1987. "Carrion Fly Community Dynamics: Patchiness, Seasonality and

505 Coexistence.” Ecological Entomology 12(3):257-66.

506 Hocking, Morgan D. and John Reynolds. 2012. "Nitrogen Uptake by Plants Subsidized by 507 Pacific Salmon Carcasses: A Hierarchical Experiment.” Canadian Journal of Forest $508 \quad$ Research 42:908-17.

509 Hocking, Morgan D. and John D. Reynolds. 2011. "Impacts of Salmon on Riparian Plant 510 Diversity." Science 331(6024):1609-12.

511 Houston, D. C. and J. E. Cooper. 1975. "The Digestive Tract of the Whiteback Griffon Vulture 512 and Its Role in Disease Transmission among Wild Ungulates." Journal of Wildlife Diseases $513 \quad 11(3): 306-13$.

514 Howard, Gary T., Bronwyn Duos, and Erin J. Watson-Horzelski. 2010. "Characterization of the 515 Soil Microbial Community Associated with the Decomposition of a Swine Carcass." 516 International Biodeterioration and Biodegradation 64(4):300-304.

517 Hyde, Embriette R., Daniel P. Haarmann, Aaron M. Lynne, Sibyl R. Bucheli, and Joseph F. 518 Petrosino. 2013. "The Living Dead: Bacterial Community Structure of a Cadaver at the 519 Onset and End of the Bloat Stage of Decomposition.” PLoS ONE 8(10):e77733.

520 Janzen, Daniel H. 1977. "Why Fruits Rot, Seeds Mold, and Meat Spoils.” The American $521 \quad$ Naturalist 111(980):691-713.

522 Jordan, Heather R., Jeffery K. Tomberlin, Thomas K. Wood, and M. Eric Benbow. 2015. 523 "Interkingdom Ecological Interactions of Carrion Decomposition.” Pp. 433-59 in Carrion 524 Ecology, Evolution, and Their Applications. 
525 Knights, Dan, Justin Kuczynski, Emily S. Charlson, Jesse Zaneveld, Michael C. Mozer, Ronald

526 G. Collman, Frederic D. Bushman, Rob Knight, and Scott T. Kelley. 2011. "Bayesian

527 Community-Wide Culture-Independent Microbial Source Tracking." Nature Methods

$528 \quad 8(9): 761-63$.

529 Kozich, James J., Sarah L. Westcott, Nielson T. Baxter, Sarah K. Highlander, and Patrick D.

530 Schloss. 2013. "Development of a Dual-Index Sequencing Strategy and Curation Pipeline

531 for Analyzing Amplicon Sequence Data on the MiSeq Illumina Sequencing Platform.”

532 Applied and Environmental Microbiology 79(17):5112-20.

533

534

535

536

537

538

539

540

541

542

543

544

545

Lau, M. K. 2013. DTK: Dunnett-Tukey-Kramer Pairwise Multiple Comparison Test Adjusted for Unequal Variances and Unequal Sample Sizes. Vol. 3.

Lee, Jae Kook, Youn Yeop Lee, Kwan Ho Park, Jeonggu Sim, Youngcheol Choi, and Sung Jae Lee. 2014. "Wohlfahrtiimonas Larvae Sp. Nov., Isolated from the Larval Gut of Hermetia Illucens (Diptera: Stratiomyidae).” Antonie van Leeuwenhoek, International Journal of General and Molecular Microbiology 105(1):15-21.

Matuszewski, Szymon, Daria Bajerlein, Szymon Konwerski, and Krzysztof Szpila. 2010. “Insect Succession and Carrion Decomposition in Selected Forests of Central Europe. Part 1: Pattern and Rate of Decomposition.” Forensic Science International 194(1-3):85-93.

McMurdie, Paul J. and Susan Holmes. 2013. "Phyloseq: An R Package for Reproducible Interactive Analysis and Graphics of Microbiome Census Data." PloS One 8(4):e61217.

McMurdie, Paul J. and Susan Holmes. 2014. "Waste Not, Want Not: Why Rarefying Microbiome Data Is Inadmissible.” PLoS Computational Biology. 
546 Metcalf, Jessica L., Laura Wegener Parfrey, Antonio Gonzalez, Christian L. Lauber, Dan

547 Knights, Gail Ackermann, Gregory C. Humphrey, Matthew J. Gebert, Will Van Treuren,

548 Donna Berg-lyons, Kyle Keepers, Yan Guo, James Bullard, Noah Fierer, David O. Carter,

549 and Rob Knight. 2013. "A Microbial Clock Provides an Accurate Estimate of the

550 Postmortem Interval in a Mouse Model System.” 1-19.

551 Michaud, Jean-Philippe and Gaétan Moreau. 2009. "Predicting the Visitation of Carcasses by

552 Carrion-Related Insects under Different Rates of Degree-Day Accumulation.” Forensic

$553 \quad$ Science International 185(1):78-83.

554 Oksanen, Jari, F. Guillaume Blanchet, Michael Friendly, Roeland Kindt, Pierre Legendre, Dan

555 McGlinn, Peter R. Minchin, R. B. O’Hara, Gavin L. Simpson, Peter Solymos, M. Henry H.

556 Stevens, Eduard Szoecs, and Helene Wagner. 2019. "Vegan: Community Ecology

557 Package.”

558 Parkinson, Rachel A., Kerith-Rae Dias, Jacqui Horswell, Paul Greenwood, Natasha Banning,

559 Mark Tibbett, and Arpad A. Vass. 2009. "Microbial Community Analysis of Human

560 Decomposition on Soil.” Pp. 379-94 in Criminal and Environmental Soil Forensics, edited

561 by K. Ritz, L. Dawson, and D. Miller. Dordrecht: Springer Netherlands.

562 Parmenter, Robert R. and James A. MacMahon. 2009. "Carrion Decomposition and Nutrient

563 Cycling in a Semiarid Shrub—Steppe Ecosystem.” Ecological Monographs 79(4):637-61.

564 Pascual, Javier, Christian von Hoermann, Ann Marie Rottler-Hoermann, Omer Nevo, Alicia

565 Geppert, Johannes Sikorski, Katharina J. Huber, Sandra Steiger, Manfred Ayasse, and Jörg

566 Overmann. 2017. "Function of Bacterial Community Dynamics in the Formation of

567 Cadaveric Semiochemicals during in Situ Carcass Decomposition.” Environmental 

Microbiology 19(8):3310-22.

569

570

Payne, Jerry A. 1965. “A Summer Carrion Study of the Baby Pig Sus Scrofa Linnaeus.” Ecology 46(5):592-602.

Pechal, Jennifer L., Tawni L. Crippen, M. Eric Benbow, Aaron M. Tarone, Scot Dowd, and Jeffery K. Tomberlin. 2014. "The Potential Use of Bacterial Community Succession in Forensics as Described by High Throughput Metagenomic Sequencing.” International Journal of Legal Medicine 128(1):193-205.

Pechal, Jennifer L., Tawni L. Crippen, Jonathan A. Cammack, Jeffery K. Tomberlin, and M. Eric Benbow. 2019. "Microbial Communities of Salmon Resource Subsidies and Associated Necrophagous Consumers during Decomposition : Potential of Cross- Ecosystem Microbial Dispersal." Food Webs 19:e00114.

Pechal, Jennifer L., Tawni L. Crippen, Aaron M. Tarone, Andrew J. Lewis, Jeffery K. Tomberlin, and M. Eric Benbow. 2013. "Microbial Community Functional Change during Vertebrate Carrion Decomposition.” PLoS ONE 8(11):1-12.

Pechal, Jennifer L., Carl J. Schmidt, Heather R. Jordan, and M. Eric Benbow. 2018. “A LargeScale Survey of the Postmortem Human Microbiome, and Its Potential to Provide Insight into the Living Health Condition.” Scientific Reports (November 2017):1-15.

Pruesse, Elmar, Jörg Peplies, and Frank Oliver Glöckner. 2012. "SINA: Accurate HighThroughput Multiple Sequence Alignment of Ribosomal RNA Genes.” Bioinformatics (Oxford, England) 28(14):1823-29.

Robinson, Mark D., Davis J. McCarthy, and Gordon K. Smyth. 2009. "EdgeR: A Bioconductor 

Bioinformatics 26(1):139-40.

591

592

593

594

595

596

597

598

599

600

601

602

603

604

605

606

607

608

609

610

Roggenbuck, Michael, Ida Bærholm Schnell, Nikolaj Blom, Jacob Bælum, Mads Frost Bertelsen, Thomas Sicheritz-Pontén, Søren Johannes Sørensen, M. Thomas P. Gilbert, Gary R. Graves, and Lars H. Hansen. 2014. “The Microbiome of New World Vultures.” Nature Communications 5:5498.

Rozen, D. E., D. J. P. Engelmoer, and P. T. Smiseth. 2008. “Antimicrobial Strategies in Burying Beetles Breeding on Carrion.” Proceedings of the National Academy of Sciences 105(46):17890-95.

Schloss, Patrick D. and Sarah L. Westcott. 2011. “Assessing and Improving Methods Used in Operational Taxonomic Unit-Based Approaches for 16S RRNA Gene Sequence Analysis.” Applied and Environmental Microbiology 77:3219-26.

Schloss, Patrick D., Sarah L. Westcott, Thomas Ryabin, Justine R. Hall, Martin Hartmann, Emily B. Hollister, Ryan A. Lesniewski, Brian B. Oakley, Donovan H. Parks, Courtney J. Robinson, Jason W. Sahl, Blaz Stres, Gerhard G. Thallinger, David J. Van Horn, and Carolyn F. Weber. 2009. "Introducing Mothur: Open Source, Platform-Independent, Community-Supported Software for Describing and Comparing Microbial Communities." Appl. Environ. Microbiol. 75:7537-7541.

Schoenly, K. G. and W. Reid. 1987. "Dynamics of Heterotrophic Succession in Carrion Arthropod Assemblages: Discrete Series or a Continuum of Change?” Oecologia 73:192202.

Shukla, Shantanu P., Heiko Vogel, David G. Heckel, Andreas Vilcinskas, and Martin 
611

612 Kaltenpoth. 2017. "Burying Beetles Regulate the Microbiome of Carcasses and Use It to Transmit a Core Microbiota to Their Offspring.” Molecular Ecology (March).

Tiegs, Scott D., Emily Y. Campbell, Peter S. Levi, Janine Rüegg, Mark Eric Benbow, Dominic T. Chaloner, Richard W. Merritt, Jennifer L. Tank, and Gary A. Lamberti. 2009. “Separating Physical Disturbance and Nutrient Enrichment Caused by Pacific Salmon in Stream Ecosystems." Freshwater Biology 54(9):1864-75.

Tiegs, Scott D., Peter S. Levi, Janine Rüegg, Dominic T. Chaloner, Jennifer L. Tank, and Gary A. Lamberti. 2011. "Ecological Effects of Live Salmon Exceed Those of Carcasses During an Annual Spawning Migration.” Ecosystems 14(4):598-614.

Tobler, Mathias. 2007. "Camera Base."

Tóth, Erika M., Peter Schumann, Andrea K. Borsodi, Zsuzsa Kéki, Attila L. Kovács, and Károly Márialigeti. 2008. "Wohlfahrtiimonas Chitiniclastica Gen. Nov., Sp. Nov., a New Gammaproteobacterium Isolated from Wohlfahrtia Magnifica (Diptera: Sarcophagidae)." International Journal of Systematic and Evolutionary Microbiology 58(4):976-81.

Weatherbee, Courtney R., Jennifer L. Pechal, and M. Eric Benbow. 2017. "Special Collection : Filth Fly - Microbe Interactions The Dynamic Maggot Mass Microbiome.” 110(1):45-53.

Wickham, Hadley. 2016. Ggplot2: Elegant Graphics for Data Analysis. Springer-Verlag New York.

Wilson, Erin E. and Elizabeth M. Wolkovich. 2011. "Scavenging: How Carnivores and Carrion Structure Communities." Trends in Ecology and Evolution 26(3):129-35.

Yang, Louie H. 2004. "Periodical Cicadas as Resource Pulses in North American Forests." 
$632 \quad$ Science 306(5701):1565-67.

633 


\section{Table $\mathbf{1}$ (on next page)}

Remarkably little scavenging activity observed at cow carcass sites.

Summary of scavenging activity per species per site, including number of individuals and minutes of scavenging activity observed. 


\begin{tabular}{|c|c|c|c|}
\hline Site & Species & Individuals & $\begin{array}{l}\text { Scavenging Duration } \\
\text { (Min) }\end{array}$ \\
\hline \multirow[t]{3}{*}{ Site 1} & Coyote & 1 & 3 \\
\hline & Turkey Vulture & 1 & 9 \\
\hline & Total & 2 & 12 \\
\hline \multirow[t]{2}{*}{ Site 2} & American badger & 1 & 1 \\
\hline & Total & 1 & 1 \\
\hline \multirow[t]{4}{*}{ Site 3} & Common Raven & 1 & 4 \\
\hline & Coyote & 1 & 1 \\
\hline & $\begin{array}{l}\text { White-tailed Antelope } \\
\text { Squirrel }\end{array}$ & 6 & 22 \\
\hline & Total & 8 & 27 \\
\hline \multirow[t]{4}{*}{ Site 4} & Common Raven & 3 & 15 \\
\hline & Kit Fox & 3 & 7 \\
\hline & Turkey Vulture & 9 & 172 \\
\hline & Total & 15 & 194 \\
\hline \multirow[t]{3}{*}{ Site 5} & Coyote & 2 & 6 \\
\hline & $\begin{array}{l}\text { White-tailed Antelope } \\
\text { Squirrel }\end{array}$ & 1 & 1 \\
\hline & Total & 3 & 7 \\
\hline \multirow[t]{2}{*}{ Site 6} & $\begin{array}{l}\text { White-tailed Antelope } \\
\text { Squirrel }\end{array}$ & 1 & 3 \\
\hline & Total & 1 & 3 \\
\hline \multirow[t]{4}{*}{ Site 7} & Common Raven & 16 & 101 \\
\hline & Coyote & 1 & 1 \\
\hline & Turkey Vulture & 4 & 85 \\
\hline & Total & 21 & 187 \\
\hline
\end{tabular}

3

4 Table 1: Summary of scavenging activity per species per site. 


\section{Table 2 (on next page)}

Microbial community compositions of cow carcasses are distinct at different stages of decomposition and are distinct from that of soil.

Statistics from AMOVA analysis of Morisita-Horn dissimilarities are shown. * = significant. 


\begin{tabular}{|l|l|l|l|}
\hline Description & Comparison & F & P-value \\
\hline Overall & All 6 sample groups & 9.30736 & $<0.001^{*}$ \\
\hline & Day 1 vs. Day 4 & 5.88847 & $0.001^{*}$ \\
\hline & Day 4 vs. Day 12 & 7.56111 & $<0.001^{*}$ \\
\hline & Day 12 vs. Day 18 & 0.406973 & 0.738 \\
\hline & Day 18 vs. Day 26 & -0.22461 & 0.978 \\
\hline & Soil vs. Day 1 & 11.173 & $0.001^{*}$ \\
\cline { 2 - 4 } & Soil vs. Day 4 & 7.47528 & $<0.001^{*}$ \\
& Soil vs. Day 12 & 14.5593 & $<0.001^{*}$ \\
\cline { 2 - 4 } & Soil vs. Day 18 & 16.8612 & $0.002^{*}$ \\
\cline { 2 - 4 } & Soil vs. Day 26 & 16.8429 & $<0.001^{*}$ \\
\hline & & & \\
\hline
\end{tabular}

2

3 Table 2: AMOVA analysis of significant differences in microbial community compositions. * = 4 significant 
Figure 1

Photographs of the decomposition progression at one of the seven sites.

The photos from this site were selected for clarity purposes, and all other sites had similar carcass composition. 

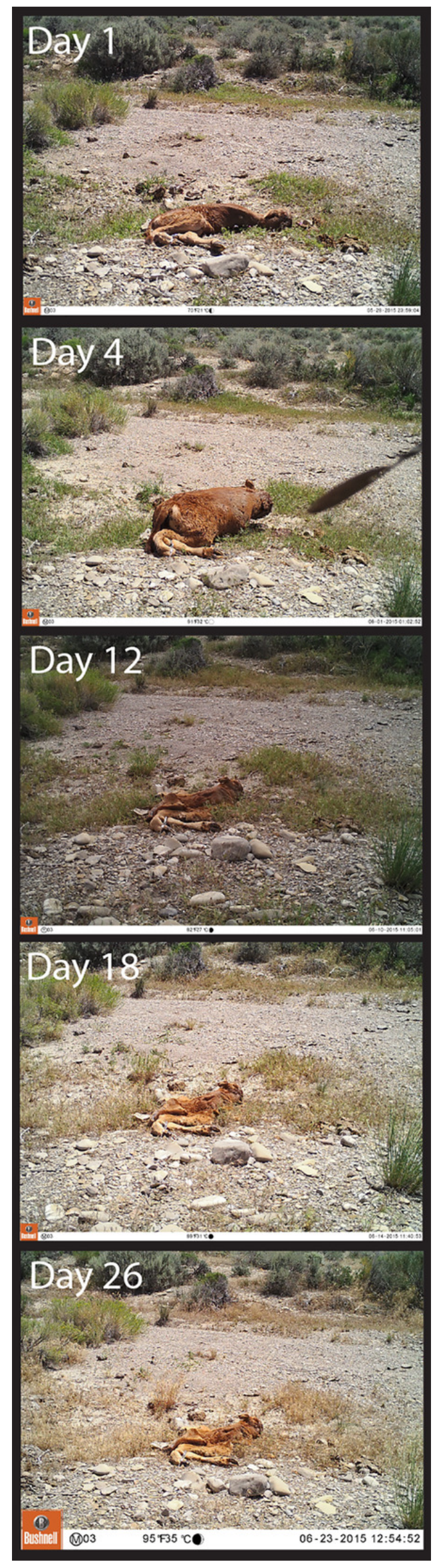

PeerJ reviewing PDF | (2019:08:40564:2:0:NEW 27 Apr 2020) 
Figure 2

Nonmetric multidimensional scaling (NMDS) plot showing bacteria community shifts associated with the stages of decomposition.

The ellipses indicate where $95 \%$ of samples within a sample period are expected to occur on the plot. 


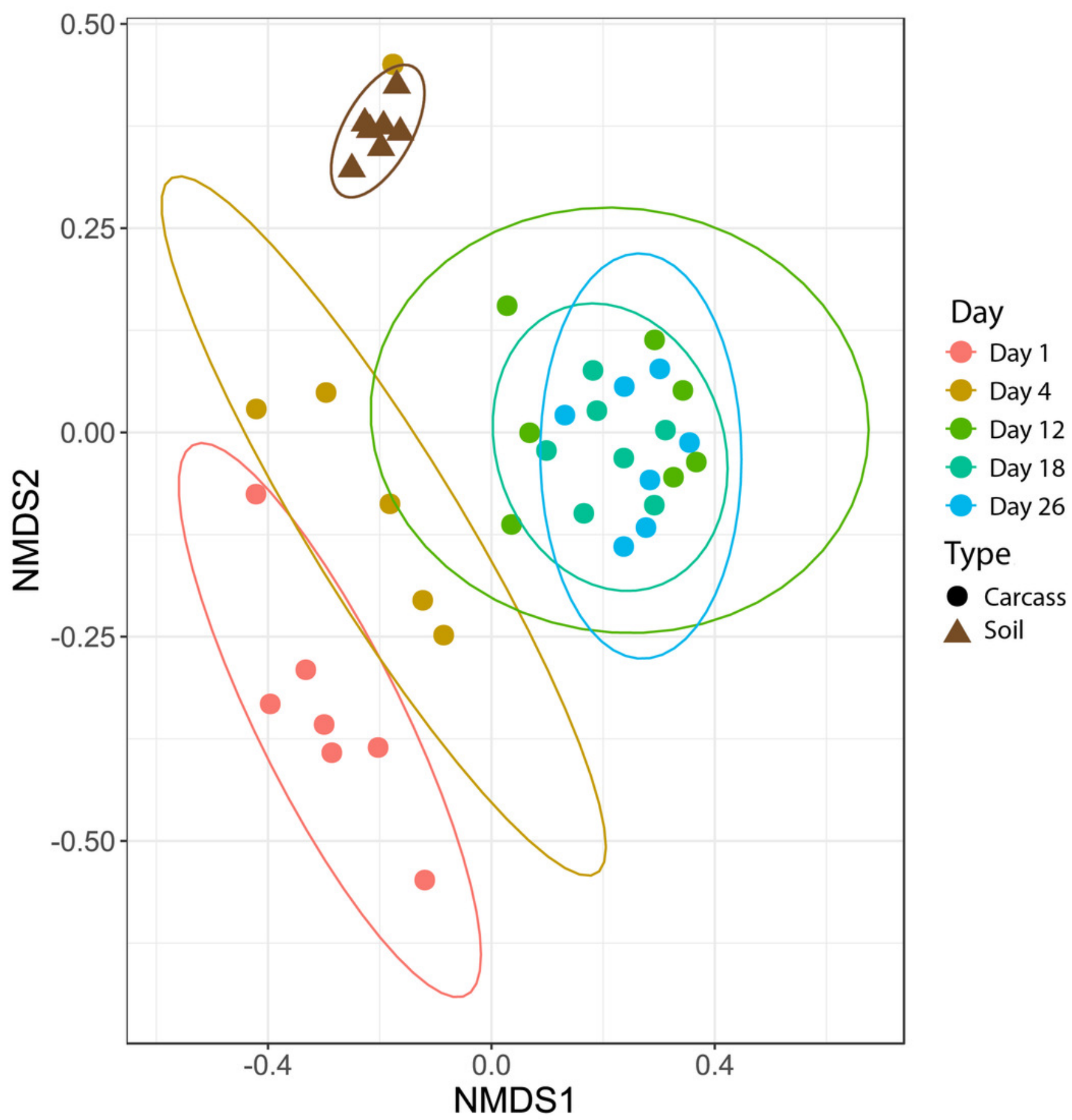


Figure 3

Abundance of genera associated with macroinvertebrates.

All genera except Myroides and Providencia exhibited abundance increases in the latter sampling periods. 


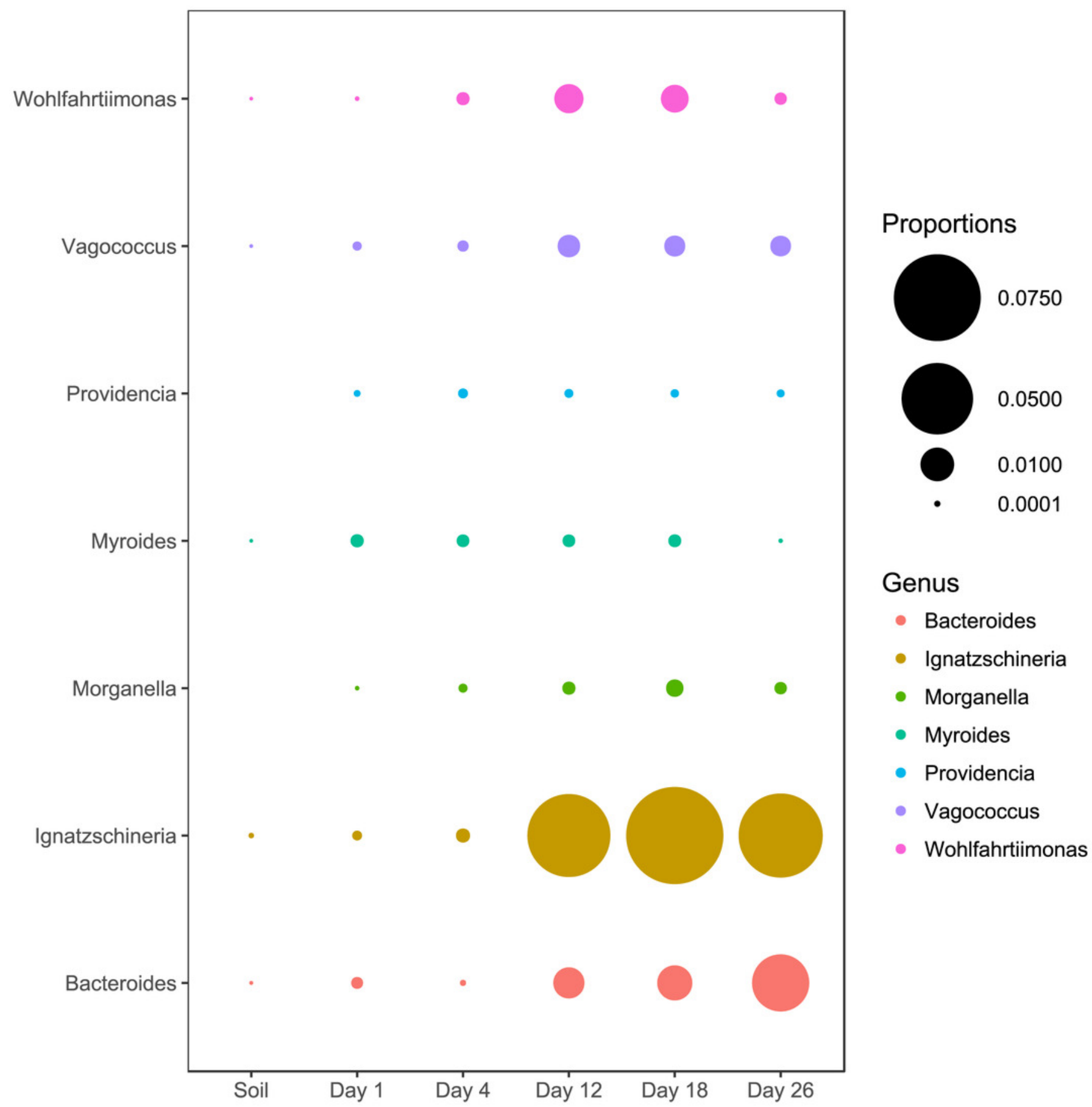




\section{Figure 4}

Bacterial communities of each stage of decomposition.

Taxa that are present throughout all stages or in soil is represented by "Ubiquitous taxa". The remain taxa are uniquely abundant within stage of decomposition. Uniquely abundant taxa that had $<1 \%$ abundance was grouped into "Low abundance taxa". 


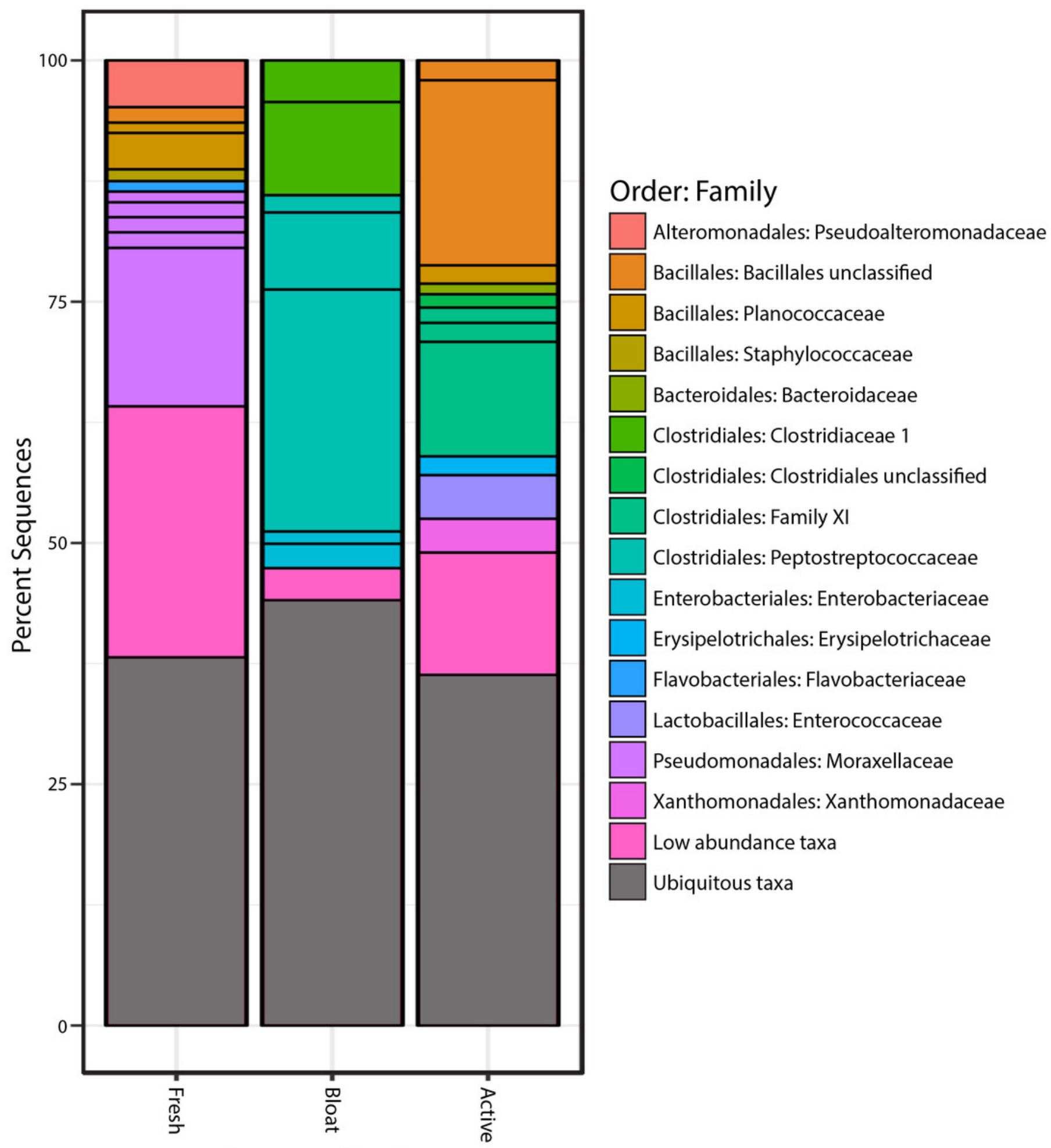

Decomposition Stage 


\section{Figure 5}

Average Shannon alpha diversity and standard error between decomposition stages and soil.

Significance of each comparison was conducted by the Dunnett-Tukey-Kramer test. All comparisons were significantly different from one another except fresh vs active decay. The red dot indicates an outlier (the sample that was contaminated by soil). ${ }^{*}=$ significant $(p<0.05)$ 


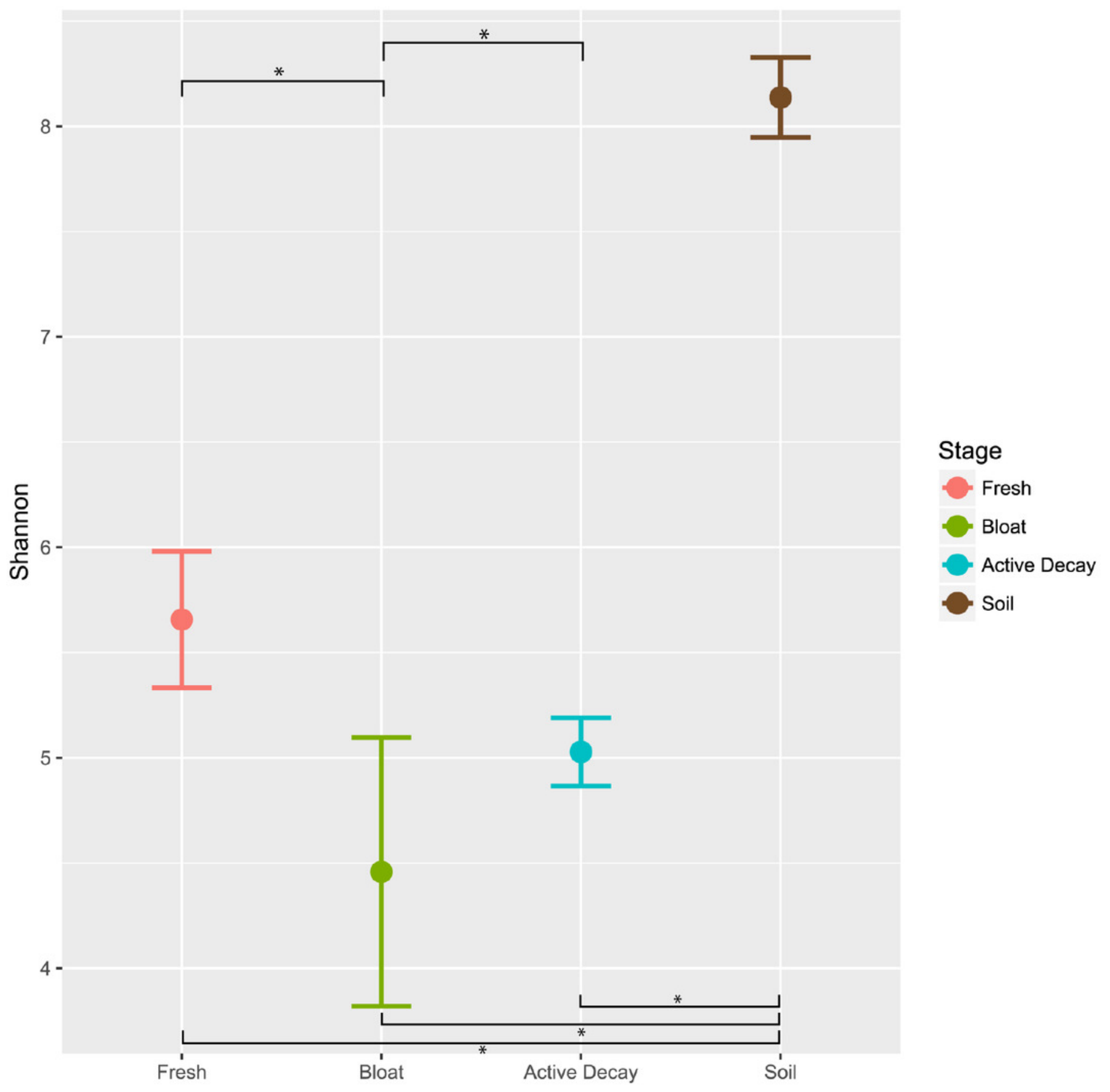

